\title{
The Bäcklund and the Galilei Invariant Transformations Constructed by Similarity Variables for Soliton Equations
}

\author{
Shunji KAWAMOTO \\ Department of Electrical and Electronic Systems, \\ University of Osaka Prefecture, 1-1 Gakuen-cho, Sakai, Osaka 593, JAPAN
}

\begin{abstract}
The Painlevé-test has been applied to checking the integrability of nonlinear PDEs, since similarity solutions of many soliton equations satisfy the Painlevé equation. As is well known, such similarity solutions can be obtained by the infinitesimal transformation, that is, the classical similarity analysis, and also the dimension of the PDEs can be reduced.

In this paper, the $\mathrm{KdV}$, the $\mathrm{mKdV}$, and the nonlinear Schrödinger equations are considered and are transformed into equations with loss and/or nonuniformity by transformations constructed on a basis of the local similarity variables. The transformations include the Bäcklund and the Galilei invariant ones. It should be noticed that the approach is applicable to other PDEs and for nonlocal similarity variables.
\end{abstract}

\section{Introduction}

From a standpoint that similarity solutions [1-3] of nonlinear PDEs satisfy the Painlevé equation $[4,5]$, the so-called Painlevé-test was proposed and has been applied to the judgement of integrability. In the classical similarity analysis, especially the following two points are characterized: If a differential equation is invariant under the transformations, (i) its order can be reduced by one, and (ii) it is possible to find similarity solutions of the equation. Tajiri and the author have shown that the KP equation and the cylindrical $\mathrm{KdV}$ equation can be reduced to the Painlevé equation $[7,8]$. Also, nonlinear PDEs reduced to the Painlevé equation have been derived through similarity solutions [9]. As an interesting example, the author has pointed out that the Harry-Dym equation can not pass the Painlevé-test, but can be rewritten into the mKdV equation by the hodograph transformation [10]. Recently, the symmetry analysis of Maxwell's equations etc. has been developed $[11,12]$. 
The aim of this paper is to construct the Bäcklund transformation (B tr.) and the Galilei invariant transformation (GI tr.) on a basis of the local similarity variables of the $\mathrm{KdV}$, the $\mathrm{mKdV}$, and the nonlinear Schrödinger (NLS) equations. First, it is shown that the GI tr. of the KdV equation is derived and the equation can be transformed to the $\mathrm{KdV}$ equation with loss and nonuniformity terms. For the NLS equation, the GI tr. and the B tr. to the wave equation describing a linearly inhomogeneous plasma are constructed and the equation for optical soliton communications with fiber loss [13]. Finally, as concluding remarks, it is summarized that the proposed approach will be practical for finding the B tr. and the GI tr. of nonlinear PDEs.

\section{The Classical Similarity Method and Transformations}

In this section, the classical similarity method and an approach to obtain the B tr. and the GI tr. are briefly explained. As a general form, we consider

$$
F\left(x, t, u, u_{x}, u_{t}, u_{x x}, \cdots \cdots\right)=0,
$$

and the infinitesimals as

$$
\begin{aligned}
x^{*} & =x+\epsilon \xi(x, t, u)+O\left(\epsilon^{2}\right), \\
t^{*} & =t+\epsilon \tau(x, t, u)+O\left(\epsilon^{2}\right), \\
u^{*} & =u+\epsilon \eta(x, t, u)+O\left(\epsilon^{2}\right),
\end{aligned}
$$

Then, eq. (1) can be transformed to

$$
F\left(x^{*}, t^{*}, u^{*}, u_{x^{*}}^{*}, u_{t^{*}}^{*}, u_{x^{*} x^{*}}^{*}, \cdots \cdots\right)=0 .
$$

Under the invariant condition on the solution surface, we have

$$
\eta(x, t, u)=\xi(x, t, u) \frac{\partial u}{\partial x}+\tau(x, t, u) \frac{\partial u}{\partial t},
$$

and the solution satisfies the characteristic equation, that is,

$$
\frac{d x}{\xi(x, t, u)}=\frac{d t}{\tau(x, t, u)}=\frac{d u}{\eta(x, t, u)}
$$

Eq. (5) gives

$$
\begin{aligned}
& \frac{d x}{d t} \equiv f_{1}(x, t, u), \\
& \frac{d u}{d t} \equiv f_{2}(x, t, u),
\end{aligned}
$$

and integrating eq. (6) yields

$$
x=g_{1}\left(t, u, C_{1}\right)
$$

with an integration constant $C_{1}$. From eq. (7), we find similarly, using a constant $C_{2}$,

$$
u=g_{2}\left(x, t, C_{2}\right) .
$$


Here, regarding $C_{1}$ and $C_{2}$ as an independent variable and the dependent one, respectively, eqs. (8) and (9) can be rewritten in the form

$$
\begin{aligned}
& C_{1} \equiv \zeta=G_{1}(x, t, u), \\
& C_{2}=C_{2}\left(C_{1}\right) \equiv f(\zeta)=G_{2}(x, t, u) .
\end{aligned}
$$

Substituting them into eq. (1), we arrive at the ODE

$$
K\left(\zeta, f, f^{\prime}, f^{\prime \prime}, \cdots \cdots\right)=0 .
$$

Moreover, from eqs. (10) and (11), if we formaly set

$$
\begin{aligned}
\zeta & \equiv X=G_{1}(x, t, u), \\
T & \equiv T(t), \\
f(\zeta) & \equiv U(X, T)=G_{2}(x, t, u), \\
u & =G(x, t, U(X, T)),
\end{aligned}
$$

thus eq.(1) becomes

$$
\tilde{F}\left(X, T, U, U_{X}, U_{T}, U_{X X}, \cdots \cdots\right)=0 .
$$

Therefore, eqs. (13)-(15) are the B tr. between eqs. (1) and (16), and the case of eq. $(1)=$ eq. (16) corresponds to the GI tr.

\section{Soliton Equations}

As examples, we treat the KdV, the mKdV and the NLS equations.

\subsection{The KdV equation}

We start from the KdV equation

$$
u_{t}+u u_{x}+\frac{1}{2} u_{x x x}=0,
$$

and find the infinitesimals as

$$
\begin{aligned}
\xi & =c_{1} x+c_{2} t+c_{3}, \\
\tau & =3 c_{1} t+c_{4}, \\
\eta & =-2 c_{1} u+c_{2},
\end{aligned}
$$

where $c_{1}, c_{2}, c_{3}$ and $c_{4}$ are arbitrary constants. So, the local similarity variables of the general case are

$$
\begin{gathered}
\zeta=\frac{x-\frac{c_{2}}{2 c_{1}} t+\frac{1}{c_{1}}\left(c_{3}-\frac{c_{2} c_{4}}{2 c_{1}}\right)}{\left(3 c_{1} t+c_{4}\right)^{1 / 3}}, \\
u(x, t)=\frac{c_{2}}{2 c_{1}}+\frac{1}{\left(3 c_{1} t+c_{4}\right)^{2 / 3}} f(\zeta) .
\end{gathered}
$$


Next, according to eqs. (13)-(15), we set the following;

$$
\begin{aligned}
X & =\frac{l x+m t+n}{(p t+q)^{1 / 3}}, \\
T & =g_{0}(t), \\
u & =r+\frac{1}{(p t+q)^{2 / 3}} U(X, T),
\end{aligned}
$$

where $l, m, n, p, q$ and $r$ are real constants, and $g_{0}(t)$ is a function of $t$ to be determined later. If we choose $g_{0}(t)=(1 / p) \ln |p t+q|$ and $m=-l r$, eq. (17) becomes the $\mathrm{KdV}$ equation with loss and nonuniformity

$$
U_{T}-\frac{2}{3} p U-\frac{1}{3} p X U_{X}+l U U_{X}+\frac{1}{2} l^{3} U_{X X X}=0 .
$$

Also, supposing $m=p=0, l=q=1, n=-r$ and $g_{0}(t)=t$ gives

$$
\begin{aligned}
X & =x-r t \\
T & =t \\
u & =r+U(X, T), \\
U_{T} & +U U_{X}+\frac{1}{2} U_{X X X}=0 .
\end{aligned}
$$

Therefore, the transformation (23) is the GI tr.

\subsection{The $\mathrm{mKdV}$ equation}

Next, for the mKdV equation

$$
u_{t}+u^{2} u_{x}+\frac{1}{2} u_{x x x}=0
$$

we get the local infinitesimals

$$
\begin{aligned}
\xi & =c_{1} x+c_{2}, \\
\tau & =3 c_{1} t+c_{3}, \\
\eta & =-c_{1} u,
\end{aligned}
$$

and the similarity variables

$$
\begin{aligned}
& \zeta=\frac{1}{\left(3 c_{1} t+c_{3}\right)^{1 / 3}}\left(x+\frac{c_{2}}{c_{1}}\right), \\
& u(x, t)=\frac{1}{\left(3 c_{1} t+c_{3}\right)^{1 / 3}} f(\zeta) .
\end{aligned}
$$

Then, by the transformation

$$
\begin{aligned}
X & =l x t^{-1 / 3}, \\
T & =q \ln |r t|, \\
u & =p t^{-1 / 3} U(X, T),
\end{aligned}
$$


we obtain

$$
U_{T}-\frac{1}{3 q}(X U)_{X}+\frac{l p^{2}}{q} U^{2} U_{X}+\frac{l^{3}}{2 q} U_{X X X}=0
$$

which has an N-soliton solution, and setting $l=p=q=1$ for simplicity gives the GI tr. as

$$
\begin{aligned}
\tilde{X} & =X+m \exp \left(-\frac{1}{3} T\right), \\
\tilde{T} & =T \\
U(X, T) & =\tilde{U}(\tilde{X}, \tilde{T}) .
\end{aligned}
$$

\subsection{The NLS equation}

The NLS equation describing the pulse propagation in optical fiber is given by

$$
i u_{t}+u_{x x}+2|u|^{2} u=0
$$

and similarly the local infinitesimals are

$$
\begin{aligned}
\xi & =c_{1} x+2 c_{2} t+c_{3}, \\
\tau & =2 c_{1} t+c_{4}, \\
\eta & =\left\{-c_{1}+i\left(c_{2} x+c_{5}\right)\right\} u .
\end{aligned}
$$

The variables can be classified into the following three cases.

(i) $c_{1}, c_{2}=0, c_{3}, c_{4}, c_{5} \neq 0$ : In this case, we have

$$
\begin{aligned}
\zeta & =c_{4} x-c_{3} t \\
u & =f(\zeta) \exp \left\{i\left(c_{4} x+\left(\frac{c_{5}}{c_{4}}-c_{3}\right) t\right)\right\},
\end{aligned}
$$

and by the $\mathrm{B}$ tr.

$$
\begin{aligned}
& X=l x+m t, \\
& T=t, \\
& u(x, t)=U(X, T) \exp \{i(p x+q t)\},
\end{aligned}
$$

eq.(32) becomes

$$
i\left\{U_{T}+(2 l p+m) U_{X}\right\}-\left(p^{2}+q\right) U+l^{2} U_{X X}+2|U|^{2} U=0 .
$$

Especially, choosing $l=1, m=-2 p$ and $q=-p^{2}$, we find eq. (36)=eq. (32) and the B tr. (35).

(ii) $c_{1}=0, c_{2} \sim c_{5} \neq 0$ : Similarly, we derive

$$
\begin{aligned}
& X=x-2 \alpha t^{2}, \\
& T=t, \\
& u(x, t)=U(X, T) \exp \left\{i 2 \alpha\left(x t-\frac{4}{3} \alpha t^{2}\right)\right\},
\end{aligned}
$$


and eq. (32) can be transformed to

$$
i U_{T}+U_{X X}+2|U|^{2} U=2 \alpha X U
$$

which is an equation for the wave in a linearly inhomogeneous plasma with cubic nonlinearity.

(iii) $c_{1} \sim c_{5} \neq 0$ : Therefore, the general case gives

$$
\begin{aligned}
X & =\frac{x+m}{\sqrt{|n t+k|}}-\frac{2 s}{n} \sqrt{|n t+k|}, \\
T & =\frac{1}{n} \ln |n t+k|, \\
u & =\frac{1}{\sqrt{|n t+k|}} U(X, T) \exp \left\{i\left(r \ln |n t+k|+s(x+m)-\frac{2 s^{2}}{n}(n t+k)+s^{2} t\right\},\right.
\end{aligned}
$$

and eq.(32) becomes

$$
i U_{T}+U_{X X}+2|U|^{2} U=i \frac{n}{2}(X U)_{X}+n r U .
$$

The first term of the r.h.s includes the fiber loss term $i(n / 2) U$.

\section{Concluding Remarks}

We have shown that the B tr. and the GI tr. can be constructed by the classical similarity analysis for soliton equations. In the sense that the $\mathrm{KdV}$, the $\mathrm{mKdV}$, and the NLS equations treated here are integrable, the resultant equations with loss and/or nonuniformity, which are physically interesting, are integrable, too. In particular, eq. (40) will be related to the soliton propagation in optical fiber with loss. Finally, it should be emphasized that this approach can be applied to other PDEs and the case of nonlocal similarity variables. 


\section{References}

[1] Ames W.F., Nonlinear Partial Differential Equations in Engineering, Academic Press, New York, Vol. I, 1965, Vol. II, 1972.

[2] Bluman G.M. and Cole J.D., Similarity Methods for Differential Equations, Springer, Berlin, 1974.

[3] Olver P.J., Applications of Lie Groups to Differential Equations, Springer, New York, 1986.

[4] Barouch E., McCoy B.M. and Wu T.T., Phys. Rev. Lett., 1973, V.31, 1409.

[5] Ablowitz M.J. and Segur H., Phys. Rev. Lett., 1977, V.38, 1103.

[6] Weiss J., Tabor M. and Carnevale G., J. Math. Phys., 1983, V.24, 522.

[7] Tajiri M. and Kawamoto S., J. Phys. Soc. Jpn., 1982, V.51, 1678.

[8] Tajiri M., Nishitani T. and Kawamoto S., J. Phys. Soc. Jpn., 1982, V.51, 2350.

[9] Kawamoto S., J. Phys. Soc. Jpn., 1983, V.52, 4059.

[10] Kawamoto S., J. Phys. Soc. Jpn., 1985, V.54, 2055.

[11] Fushchych W.I. and Nikitin A.G., Symmetries of Maxwell's Equations, D. Reidel Publishing Company, 1987.

[12] Fushchych W., Shtelen W. and Serov N., Symmetry Analysis and Exact Solutions of Equations of Nonlinear Mathematical Physics, Dordrecht, Kluwer Academic Publishers, 1993.

[13] Hasegawa A. and Kodama Y., Solitons in Optical Communications, Oxford University Press, New York, 1995. 